\title{
Ten years of prophylactic treatment with fresh-frozen plasma in a child with chronic relapsing thrombotic thrombocytopenic purpura as a result of a congenital deficiency of von Willebrand factor-cleaving protease
}

\author{
José Barbot, ${ }^{1}$ Elísio Costa, ${ }^{1}$ Marisol Guerra, ${ }^{1}$ Maria S. Barreirinho, ${ }^{1}$ Pratima Isvarlal, ${ }^{1}$ \\ Rodolfo Robles, ${ }^{2}$ Helena E. Gerritsen, ${ }^{2}$ Bernhard Lämmle ${ }^{2}$ and Miha Furlan ${ }^{2}{ }^{1}$ Serviço de Hematologia do \\ Hospital de Crianças Maria Pia, Porto, Portugal, and ${ }^{2}$ Central Haematology Laboratory, University Hospital, Inselspital, \\ Berne, Switzerland
}

Received 4 December 2000; accepted for publication 22 January 2001

\begin{abstract}
Summary. We report the results of 10 years of prophylactic fresh-frozen plasma (FFP) infusion therapy in a 14-year-old girl with chronic relapsing thrombotic thrombocytopenic purpura (TTP), in whom a severe congenital von Willebrand factor (VWF)-cleaving protease deficiency has been documented. Severe haemolytic crises triggered by infections were prevented and her present renal and neurological functions have been preserved. Sequential measurements of
\end{abstract}

protease activity and platelet count after FFP infusion led us to conclude tentatively that $5 \%$ may be sufficient to degrade very large and adhesive VWF multimers.

Keywords: VWF-cleaving protease, deficiency, fresh-frozen plasma, chronic haemolytic anaemia, thrombotic thrombocytopenic purpura.
Thrombotic thrombocytopenic purpura (TTP) is a potentially fatal disease, characterized by widespread platelet thrombi in the microcirculation. Excessive intravascular platelet agglutination seems to be associated with massive release from endothelial cells of unusually large von Willebrand factor (VWF) multimers and/or their impaired degradation to smaller non-agglutinating molecular forms by a specific VWF-cleaving protease. Congenital deficiency of this protease was found in patients with familial forms of the disease while a protease inhibitor has been observed in acquired TTP (Furlan et al, 1998; Tsai \& Lian, 1998).

We describe a patient with congenital deficiency of VWFcleaving protease and the follow-up after 10 years of prophylactic treatment with fresh-frozen plasma (FFP).

\section{CASE REPORT}

The patient was a 14-year-old Caucasian female born in northern Portugal of first-degree cousins (Fig 1A). She

Correspondence: José Barbot, Serviço de Hematologia, Hospital de Crianças Maria Pia, Rua da Boavista, 827, 4050-111 Porto, Portugal. E-mail: hematologica@hmaria.min-saude.pt developed hyperbilirubinaemia within the first $12 \mathrm{~h}$ of life, requiring $48 \mathrm{~h}$ of phototherapy. She was referred to our hospital at 24 months of age for haemolytic anaemia and thrombocytopenia triggered by an infectious event. She was discharged after improvement without an aetiological diagnosis. Subsequently, she led a normal life without pallor or haemorrhagic symptoms. Nevertheless, her haematological findings were not normal. A well-compensated haemolytic anaemia and thrombocytopenia were persistently present. Neither morphological red cell abnormalities nor evidence of immunomediated red cell or platelet destruction was found.

At the age of 3 years, the patient was admitted again. She was seriously ill with fever, prostration, vomiting and dark urine. Her haemoglobin level had dropped to $4.8 \mathrm{~g} / \mathrm{dl}$ with a marked reticulocytosis. The platelet count was $23 \times 10^{9} / \mathrm{l}$ and the lactate dehydrogenase level was $2260 \mathrm{IU} / \mathrm{l}$. The presence of numerous schistocytes and microspherocytes in the blood film and massive haemoglobinaemia and haemoglobinuria indicated the intravascular and microangiopathic nature of the haemolytic process. Mild proteinuria was detected but the renal function was preserved. Coagulation screening was normal. No neurological impairment was observed. She was discharged after red cell 


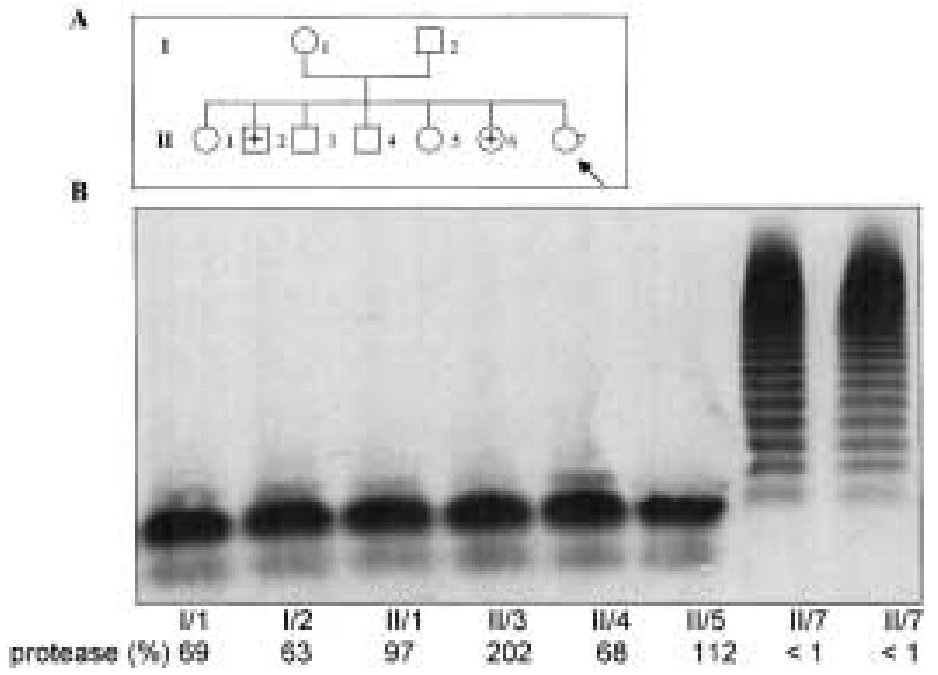

Fig 1. (A) Family pedigree. The arrow indicates the patient with chronic relapsing TTP. Two siblings of the proposita (family members II/2 and II/6) had died at the ages of $4 \mathrm{~d}$ and 5 months respectively. (B) Results of VWF-cleaving protease assay. The immunoblot shows no degradation of VWF substrate in the presence of diluted plasma samples from the proposita, whereas all members of her family had VWF-cleaving protease activity $>50 \%$. Blood samples of the patient were collected on two occasions. The protease activities as measured by the collagen binding assay are given under each lane. transfusion and antibiotic therapy, with the diagnosis of haemolytic uraemic syndrome (HUS)/TTP.

Thirty days later she was readmitted with a similar relapse $(\mathrm{Hb} 5.8 \mathrm{~g} / \mathrm{dl})$. We decided to transfuse FFP and the response was dramatic. Three days later she had the first normal platelet count in the course of her disease. The haemolytic process was arrested and a gradual $\mathrm{Hb}$ recovery was observed. After a subsequent relapse, a prophylactic regimen with regular infusions of FFP was instituted. Initially, FFP from a single donor was used, but later a commercial virus-inactivated FFP was used. The follow-up was indicative of the beneficial effects of plasma infusions. The only significant crisis occurred once in the fourth week after FFP infusion. This event led us to reduce the intervals between infusions from 4 weeks to 3 weeks. We found that even quite small volumes of FFP were able to prevent crises. In the beginning, we transfused $10 \mathrm{ml} / \mathrm{kg}$, but did not use more than one unit of FFP when the patient exceeded $20 \mathrm{~kg}$ of body weight. Presently, aged 14 years and after 10 years of this regimen, the patient has a normal physical and intellectual development. The renal and neurological functions have been preserved.

The activities of VWF-cleaving protease in the proposita and the members of her family are shown in Fig 1B. The protease assay was performed by multimer analysis of VWF substrate following incubation with diluted test plasma sample (Furlan et al, 1997, 1999) and by measuring binding of degraded VWF to collagen (Gerritsen et al, 1999). The protease activity in our patient was less than $1 \%$ on two occasions, whereas the immunoblot assay showed normal activities $(>50 \%)$ in all four examined siblings as well as in both parents. As there was no protease inhibitor detectable in our patient, this indicated that she had severe constitutional deficiency of VWF-cleaving protease. The protease activity was also measured using the collagen binding assay that is better suited for discriminating activities in the normal range than the immunoblotting method (Gerritsen et al, 1999). Results of the latter assay showed $60-70 \%$ protease activity in both parents and in one brother, whereas two sisters had about 100\% and one brother even $200 \%$ activity.

The time-course of protease activity and platelet count after a recent prophylactic FFP infusion (August 2000) is shown in Fig 2. From the volume of FFP $(200 \mathrm{ml})$ and the body weight $(56 \mathrm{~kg})$ of the patient, an initial protease activity of about $8 \%$ was expected. The measured activity immediately after FFP infusion was 13\%, suggesting a protease recovery of more than $100 \%$ and, thus, the absence of a circulating protease inhibitor. Protease activity declined over the next few days (Fig 2) and was less than $2 \%$ on $\mathrm{d} 7$, but could not be quantified accurately. The estimated halflife of VWF-cleaving protease activity was $2 \cdot 6 \mathrm{~d}$. Normal platelet count was observed during the second week after plasma infusion.

\section{DISCUSSION}

The debate about TTP and HUS being either two different clinical entities or two different forms of a single disease has still not abated. The classic criteria for distinguishing between these two syndromes are the predominance of neurological symptoms in TTP and renal failure in HUS. However, clinical findings and laboratory data overlap in patients with TTP or HUS. Neurological involvement can be observed in patients with the diagnosis of HUS and a large proportion of patients classified as having TTP show renal failure (Thompson et al, 1992).

Schulman et al (1960) reported a case very similar to that presented here. Upshaw (1978) described another similar case and proposed that this patient, whose clinical course had begun in infancy, probably had a chronic congenital form of TTP. Moake et al (1982) studied Schulman's patient who had relapsing episodes of thrombocytopenia, intravascular haemolysis and neurological disturbances unless she was given infusions of FFP. They reported the presence in her plasma of unusually large VWF multimers. Recently, a constitutional deficiency of VWF-cleaving protease has been 


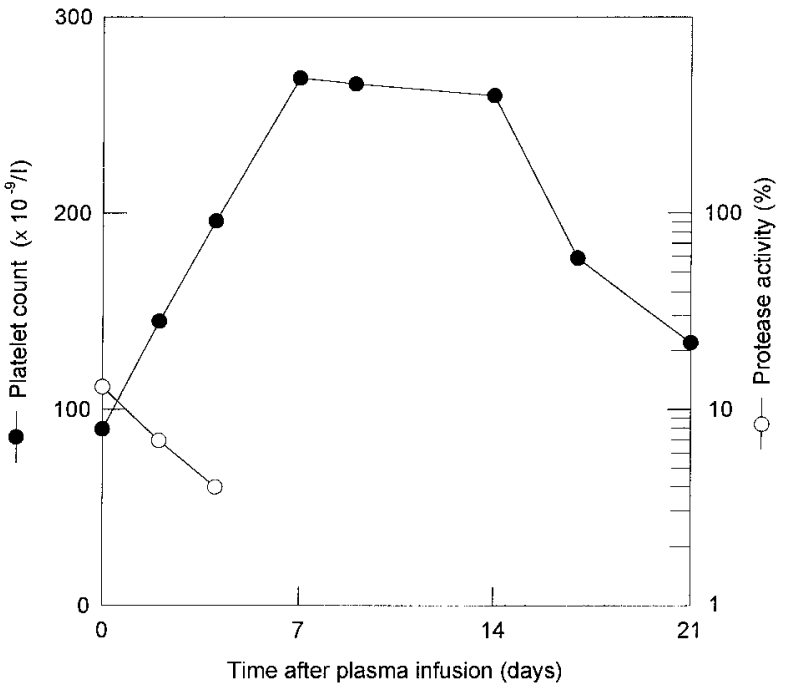

Fig 2. Platelet count and activity of VWF-cleaving protease following prophylactic infusion of $200 \mathrm{ml} \mathrm{FFP}(3.6 \mathrm{ml} / \mathrm{kg}$ body weight).

found in several patients with familial TTP (Furlan et al, 1997, 1998; Häberle et al, 1999).

Both parents and four of the six siblings of our patient were clinically asymptomatic, but the consanguinity of the parents suggested that the disease was inherited in an autosomal recessive manner. Two siblings had died at the ages of $4 \mathrm{~d}$ (with neonatal hyperbilirubinaemia) and 5 months (with a diagnosis of meningitis) respectively. Results of the VWF-cleaving protease assay showed a complete protease deficiency in proposita whereas her parents and one brother had $60-70 \%$ protease activity, suggesting that these asymptomatic family members are heterozygous for the protease deficiency.

Platelet count and activity of VWF-cleaving protease during a recent FFP infusion cycle are shown in Fig 2. The maximum platelet count was found between $\mathrm{d} 7$ and d 14. Subsequently, the platelet count decreased to about $120 \times 10^{9} / \mathrm{l}$ and the prophylactic FFP cycle was repeated. This result agrees with the report of Schulman et al (1960) who observed the highest platelet counts on d 9-11 after a single infusion of $125 \mathrm{ml} \mathrm{FFP.} \mathrm{In} \mathrm{the} \mathrm{study} \mathrm{of} \mathrm{Upshaw}$ (1978), the maximum response to two units of FFP was also evident 9-10 d after plasma infusion. The concentration of VWF-cleaving protease immediately after FFP infusion in our patient was about $13 \%$. Full recovery of infused protease and its half-life of $2.6 \mathrm{~d}$ in circulating plasma are in agreement with the recoveries and half-lives of VWFcleaving protease observed in two brothers with constitutional VWF-cleaving protease deficiency (Furlan et al, 1999). It is remarkable that the platelet count still increased until d 7 when the activity of VWF-cleaving protease had fallen below 5\%. We conclude from these observations that $5 \%$ protease activity may be sufficient to cleave the constitutionally secreted unusually large VWF multimers and prevent formation of platelet microthrombi, a characteristic of TTP.
FFP infusion was found to be extremely effective in reversing crises in our patient and in avoiding relapses, if administered regularly. We considered the benefit of this prophylactic approach against the potential harmful effects, namely the possibility of transmission of viral diseases or the development of alloantibodies against the lacking factor in the patient's plasma. After 160 FFP units transfused, there has been no clinical or serological evidence of viral disease or of a protease inhibitor. The asymptomatic course of the disease after institution of plasma therapy and the preservation of neurological and renal functions in our patient suggest that prophylactic treatment with FFP is an adequate approach to the management of the congenital form of TTP until a purified or recombinant protease becomes available.

\section{REFERENCES}

Furlan, M., Robles, R., Solenthaler, M., Wassmer, M., Sandoz, P. \& Lämmle, B. (1997) Deficiency of von Willebrand factor-cleaving protease in chronic relapsing thrombotic thrombocytopenic purpura. Blood, 89, 3097-3103.

Furlan, M., Robles, R., Galbusera, M., Remuzzi, G., Kyrle, P.A., Brenner, B., Krause, M., Scharrer, I., Aumann, V., Mittler, U., Solenthaler, M. \& Lämmle, B. (1998) von Willebrand factorcleaving protease in thrombotic thrombocytopenic purpura and the hemolytic-uremic syndrome. New England Journal of Medicine, 339, 1579-1584.

Furlan, M., Robles, R., Morselli, B., Sandoz, P. \& Lämmle, B. (1999) Recovery and half-life of von Willebrand factor-cleaving protease after plasma therapy in patients with thrombotic thrombocytopenic purpura. Thrombosis and Haemostasis, 81, 8-13.

Gerritsen, H.E., Turecek, P.L., Schwarz, H.P., Lämmle, B. \& Furlan, M. (1999) Assay of von Willebrand factor (vWF)-cleaving protease based on decreased collagen binding affinity of degraded vWF: a tool for the diagnosis of thrombotic thrombocytopenic purpura. Thrombosis and Haemostasis, 82, 1386-1389.

Häberle, J., Kehrel, B., Ritter, J., Jürgens, H., Lämmle, B. \& Furlan, M. (1999) New strategies in diagnosis and treatment of thrombotic thrombocytopenic purpura: case report and review. European Journal of Pediatrics, 158, 883-887.

Moake, J.L., Rudy, C.K., Troll, J.H., Weinstein, M.J., Colannino, N.M., Azocar, J., Seder, R.H., Hong, S.L. \& Deykin, D. (1982) Unusually large plasma factor VIII: von Willebrand factor multimers in chronic relapsing thrombotic thrombocytopenic purpura. New England Journal of Medicine, 307, 1432-1435.

Schulman, I., Pierce, M., Lukens, A. \& Currimbhoy, Z. (1960) Studies on thombopoiesis I. A factor in normal human plasma required for platelet production; chronic thrombocytopenia due to its deficiency. Blood, 16, 943-957.

Thompson, C.E., Damon, L.E., Ries, C.A. \& Linker, C.A. (1992) Thrombotic microangiopathies in the 1980s: clinical features, response to treatment, and the impact of the human immunodeficiency virus epidemic. Blood, 80, 1890-1895.

Tsai, H. \& Lian, E.C. (1998) Antibodies to von Willebrand factorcleaving protease in acute thrombotic thrombocytopenic purpura. New England Journal of Medicine, 339, 1585-1594.

Upshaw, J.D. (1978) Congenital deficiency of a factor in normal plasma that reverses microangiopathic hemolysis and thrombocytopenia. New England Journal of Medicine, 298, 1350-1352. 